\title{
Nuclear magnetic resonance spectroscopy reveals metabolic changes in living cardiomyocytes after low doses of ionizing radiation
}

\author{
Michalina Gramatyka, Agnieszka Skorupa ${ }^{\bowtie}$ and Maria Sokół \\ Department of Medical Physics, Maria Skłodowska-Curie Memorial Cancer Center and Institute of Oncology, Gliwice Branch, Gliwice, Poland
}

Several lines of evidence indicate that exposure of heart to ionizing radiation increases the risk of cardiotoxicity manifested by heart dysfunction and cardiovascular diseases. It was initially believed that the heart is an organ relatively resistant to radiation. Currently, however, it is suspected that even low doses of radiation ( $<2 \mathrm{~Gy})$ may have a negative impact on the cardiovascular system. Cardiotoxicity of ionizing radiation is associated with metabolic changes observed in cardiac cells injured by radiation. In this study, we used human cardiomyocytes as a model system, and studied their metabolic response to radiation using high-resolution magic angle spinning nuclear magnetic resonance techniques (HR-MAS NMR). Human cardiomyocytes cultured in vitro were exposed to ionizing radiation and their survival was assessed by clonogenic assay. Changes in apoptosis intensity and cell cycle distribution after the irradiation were measured as well. NMR spectra of cardiomyocytes were acquired using Bruker Avance $400 \mathrm{MHz}$ spectrometer at a spinning rate of $3200 \mathrm{~Hz}$. Survival of cardiomyocytes after NMR experiments was assessed by the Trypan blue exclusion assay. Exposure of cardiomyocytes to small doses of ionizing radiation had no effect on cell proliferation potential and intensity of cell death. However, analysis of metabolic profiles revealed changes in lipids, threonine, glycine, glycerophosphocholine, choline, valine, isoleucine, glutamate, reduced glutathione and taurine metabolism. The results of this study showed that ionizing radiation affects metabolic profiles of cardiomyocytes even at low doses, which potentially have no effect on cell viability.

Key words: ionizing radiation, metabolomics, HR MAS NMR, cardiotoxicity

Received: 05 February, 2018; revised: 23 April, 2018; accepted: 18 May, 2018; available on-line: 15 June, 2018

e-mail: agnieszka.skorupa@io.gliwice.pl

Abbreviations: Ala, alanine; Cho, choline; CPMG, Carr-Purcell-Meiboom-Gill; Cr, creatine; Glu, glutamate; Gly, glycine; GPC, glycerophosphocholine; GSH, glutathione reduced; HR-MAS NMR, highresolution magic angle spinning nuclear magnetic resonance spectroscopy; hTau, hypotaurine; lle, isoleucine; IN integral normalization; Lac, lactate; PC, phosphocholine; PCr, phosphocreatine; PTC phosphatidylcholine; PQN, probability quotient normalization; Tau, taurine; Thr, threonine; Val, valine

\section{INTRODUCTION}

Exposure of human body to ionizing radiation can cause, among others, bone marrow suppression (myelotoxicity) or cardiovascular system disorders - so-called cardiotoxicity. Reports about cardiotoxicity of ionizing radiation refer usually to relatively high doses delivered to the heart, while the negative impact of low doses (cumulative dose below 5 Gy), despite numerous studies, remains controversial and unexplained (Shimizu et al., 2010; Bouillon et al., 2011; Little et al., 2012; Kwon et al., 2014). However, the findings of the CARDIORISK collaborative research project suggest that although low-dose radiation does not per se induce atherosclerotic changes, it may indirectly affect the stress response of the heart (http://cordis.europa.eu/result/rcn/56094_ en.html).

The problem of cardiotoxicity of low-dose radiation becomes more serious because there is a high risk of exposure (not only from medical uses of radiation but also from such sources as radioactive elements, nuclear plants or cosmic radiation) and because of increasing life expectancy, which allows the revealing of long-term radiation effects (Little et al., 2012; Darby et al., 2013; Evans et al., 2015). Some researchers suggest that such low-dose exposures are harmless to the heart and unlikely to increase cardiac mortality (Prosnitz et al., 2007). However, there is a growing number of reports on the impact of low radiation doses on the frequency of heart diseases (Little et al., 2012; Darby et al., 2013; Monceau et al., 2013; Boerma et al., 2016; Delp et al., 2016). For instance, an association between lower dose exposures and late-occurring circulatory disease was recently suspected in the Japanese atomic bomb survivors and in various occupationally exposed cohorts (Little et al., 2015). These studies indicate that even low doses (below 4 Gy) may increase the risk of the cardiac event (usually ischemic heart disease), although the time between the exposure and the occurrence of clinical symptoms can be very long. Moreover, the cardiotoxicity mechanisms in case of low-dose radiation may be different from that of highdose (Little et al., 2012). These cardiotoxic effects are often progressive and irreversible, emphasizing a need for effective prevention and treatment to reduce or avoid the cardiotoxicity.

Detection and characterization of molecular features of harmful changes occurring in the heart in response to ionizing radiation are expected to enable identification of individuals at increased risk of cardiovascular disorders, and to take steps to prevent them. One of the most effective methods to detect early changes in cells in response to stress is to explore their metabolome. The metabolome is the whole qualitative and quantitative composition of the metabolites present in the biological system in certain physiological conditions. It is the final result of genome and transcriptome expression, and it seems most closely relevant to the phenotype (Eckhart et al., 2012; Rhee \& Gerszten, 2012). The levels of some metabolites are altered by various pathological states and the changes in the metabolic profile can be observed 
long before the clinical symptoms develop. What is more important, different metabolites may change depending on the toxic agent (Barba \& Garcia-Dorado, 2012).

Several studies showed that various cardiac disorders are represented by different metabolic biomarkers or sets of biomarker metabolites (Nakae et al., 2003; Faller et al., 2013). At the same time, the exact mechanism of cardiac cell response to ionizing radiation (especially in case of low doses) is unknown. To address this topic we conducted a study aimed at characterization of heart cells metabolic profile and changes therein after exposure to low-dose radiation. We applied proton HR-MAS NMR (High-Resolution Magic Angle Spinning Nuclear Magnetic Resonance) spectroscopic technique enabling noninvasive detection of metabolic changes in living cells without the need for metabolite extraction.

\section{MATERIALS AND METHODS}

Cell line and materials. Human cardiomyocytes were obtained from Celprogen (1311001-09) and cultured in flasks covered with fibronectin. Cells were cultured in DMEM/F12 medium supplemented with 10\% FBS, $2 \mathrm{mM}$ L-glutamine and antibiotics, in $37^{\circ} \mathrm{C}$ humidified atmosphere containing $5 \% \mathrm{CO}_{2}$. Irradiation of cells was performed using CLINAC 600 linear accelerator with 6MV photons and $1 \mathrm{~Gy} / \mathrm{min}$ dose rate.

Clonogenic assay. Cells were seeded into 6-well plates at a density of 1000 cells per well and irradiated with single doses of $1,2,4$ or $8 \mathrm{~Gy}$, then cultured in fresh medium for 7 days. The colonies were stained with a solution containing $0.5 \%$ crystal violet and $50 \%$ methanol, rinsed with water to remove an excess of the dye, and then the number of colonies was counted.

Cell cycle analysis. To characterize the distribution of the cell cycle phases control and irradiated cells were harvested by trypsinization and fixed with $70 \%$ ethanol. DNA in the collected cells was stained with a solution containing $1 \mathrm{ng} / \mathrm{ml}$ DAPI and $0.1 \mu \mathrm{g} / \mathrm{ml}$ RNase in PBS and then analyzed by flow cytometry using FACSCanto cytometer (Becton Dickinson).

HR-MAS NMR analysis. To characterize the metabolic changes occurring in cardiomyocytes after the irradiation HR-MAS NMR spectra of the irradiated living cells were compared with those of the control, non-irradiated cells. 48 hours after the irradiation with a single dose of 2 Gy the control and irradiated cells were harvested by trypsinization, washed in PBS, centrifuged and suspended in $20 \% \mathrm{D}_{2} \mathrm{O}$ in PBS. Solutions containing about $8 \times 10^{6}$ living cells were packed into disposable 30 $\mu \mathrm{L}$ inserts and placed in HR-MAS rotors. Three sample preparations were evaluated for each group.

${ }^{1} \mathrm{H}$ NMR spectra were acquired on a $400 \mathrm{MHz}$ Bruker Avance spectrometer at room temperature and at a spinning rate of $3200 \mathrm{~Hz}$. The total time for sample preparation and data acquisition was restricted to $1.5 \mathrm{~h}$ to prevent sample degradation. Cell survival after the NMR experiments was assessed using the Trypan blue exclusion assay. For each sample the following HR-MAS NMR experiments were performed:

1D Carr-Purcell-Meiboom-Gill (CPMG, pulse sequence: cpmgpr1D). Acquisition parameters: 256 scans, $65 \mathrm{k}$ data points, spectral width $20 \mathrm{ppm}$, relaxation delay $4 \mathrm{~s}$, acquisition time $4.08 \mathrm{~s}$, total echo time $90 \mathrm{~ms}$. Processing: the spectra were apodized with an exponential function $(0.3 \mathrm{~Hz})$, Fourier-transformed, phased and baseline corrected. The sequence utilized transverse relaxation time filter to suppress macromolecular resonances and to enhance the signal of low molecular weight metabolites.

2D J-resolved (pulse sequence: jresgpprqf). Acquisition parameters: $8 \mathrm{k}$ time domain data points, 40 increments, 8 scans per increment, spectral width $=20 \mathrm{ppm}$ (F2) and $78.2 \mathrm{~Hz}$ (F1), relaxation delay 2s. Processing: The spectra were tilted by $45^{\circ}$, baseline corrected and symmetrized about $\mathrm{J}=0 \mathrm{~Hz}$ line. $1 \mathrm{D}$ projections of $2 \mathrm{D}$ J-resolved spectra onto chemical shift axis ( $\mathrm{p}$-Jres) were calculated.

1D Diffusion-edited (pulse sequence: ledbpgp2s1dpr). Acquisition parameters: 64 scans, 65k data points, spectral width $30 \mathrm{ppm}, \Delta=200 \mathrm{~ms}, \delta=2 \times 2 \mathrm{~ms}$, eddy current delay $5 \mathrm{~ms}$, sine-shaped gradient with $36 \mathrm{G} / \mathrm{cm}$ followed by a $200 \mu$ s delay for gradient recovery. Processing: the spectra were apodized with an exponential function $(2 \mathrm{~Hz})$, Fourier-transformed, phased and baseline corrected. Low molecular weight metabolites were attenuated in relation to the macromolecular ones in the diffusion edited spectra.

The metabolites were identified based on J-resolved spectra and by comparing their chemical shifts with those for the standard compounds from Chenomx NMR Suite Professional database (Chenomx Inc., Edmonton, Canada). The 2D J-resolved technique is well suited for facilitation of metabolite identification in HR-MAS NMR studies of living cells because of relatively short acquisition time (about $18 \mathrm{~min}$ ) in comparison to the other 2D techniques (COSY, TOCSY, HSQC).

$1 \mathrm{D}$ projections of $2 \mathrm{D}$ J-resolved spectra onto chemical shift axis (p-Jres) were chosen for determination of signal integrals corresponding to low molecular weight metabolites. These spectra do not show multiplicity and the resolution of signals is improved in comparison to CPMG spectra (Ludwig \&Viant, 2010). Integration was performed for the highest and the most isolated signal arising from a particular metabolite in order to minimize the errors from the spectral overlapping. However, the remaining signals of the metabolites exhibited similar trends. The changes detected in p-Jres spectra were also apparent in CPMG spectra. Lipid signals were evaluated in diffusion-weighted spectra.

HR-MAS NMR spectra normalization. In order to detect only biologically meaningful changes in metabolite concentrations that may be obscured by experimental inter-sample variance lowering the statistical power of the analysis, the NMR data were preprocessed by two state-of-the-art normalization protocols independently: the commonly used integral (or total area) normalization (IN) (Craig, 2006) and the probabilistic quotient normalization (PQN), the method claimed to be more robust and more accurate than the widespread integral normalization (Dieterle, 2006). PQN is based on the hypothesis that a majority of molecule concentrations remain unchanged across the samples and the spectra are compared to a reference sample (the median spectrum). Such approach mitigates scaling artifacts resulting from the total area normalization since the latter is prone to the influence of highly abundant signals.

The normalization operations, applied to each spectrum, make the data from all samples directly comparable to each other - by taking into account the variations of the overall metabolic concentrations due to biological and technical reasons.

In case of IN the spectral range from 0.8 to $4.62 \mathrm{ppm}$ was integrated for each spectrum and this integral value was used as a scaling factor. The PQN approach started with a normalization of each spectrum to the total area within the range from 0.8 to $4.62 \mathrm{ppm}$. Then, the 


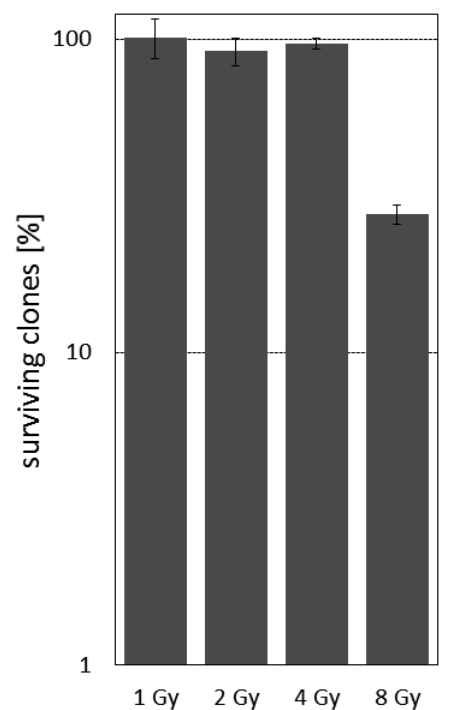

Figure 1. The influence of ionizing radiation on clonogenic survival of cardiomyocytes. Numbers of surviving clones are presented in relation to untreated controls $(100 \%)$.

median across all of the spectra was taken as the reference spectrum and the ratio of all peaks between a given spectrum and the reference spectrum was calculated. The median of these ratios in a sample was the final division factor.

\section{RESULTS}

In this work, we assessed the hypothetical effect of ionizing radiation on the metabolic profile of living hu- man cardiomyocytes cultured in vitro. In the pilot experiments, we tested several doses of ionizing radiation (up to $8 \mathrm{~Gy}$ ) to examine the influence of various radiation doses on the survival of cardiac cells and to identify the threshold dose - the smallest exposure level that will cause a specific and measured effect in the cellular tests.

The viability of cardiomyocytes treated with ionizing radiation was assessed with the clonogenic assay. Visible reduction of cell viability (by more than 50\%) was observed only after the highest radiation dose (8 Gy), which indicates a high radioresistance of cardiomyocytes (Fig. 1). Nevertheless, a lower, more clinically relevant dose (2 Gy) was selected for further experiments.

The potential effect of ionizing radiation on the blockade of cell cycle progression was assessed by flow cytometry-based analysis of the cell cycle phases distribution. The analysis revealed that exposure to $2 \mathrm{~Gy}$ induced a prolonged block in the G2/M phase, which was still detectable 72 hours after the end of the treatment (Fig. 2); the ratios of G2/G1 in the control and radiation-treated cell population were 0.23 and 0.40 , respectively. At 24 and 48 hours after irradiation, ionizing radiation increased the number of apoptotic cells (detected here as subG1 subpopulation) to $8.1 \%$ and $5.0 \%$ of the whole population respectively. Such increase was no longer detectable 72 hours after irradiation.

The metabolic status of the irradiated cells was observed using the NMR-based approach as the spectroscopic methods provide an insight into living cells without disturbing the cellular organization. ${ }^{1} \mathrm{H}$ HR-MAS NMR study of cardiomyocytes revealed metabolic changes in cells exposed to ionizing radiation at a dose of 2 Gy and, as revealed from the vitality tests, the observed changes were not due to vitality decline (Fig. 1). Figures 3,4 and 5 show the representative CPMG, p-Jres, and diffusion edited spectra, respectively. In order to evalu

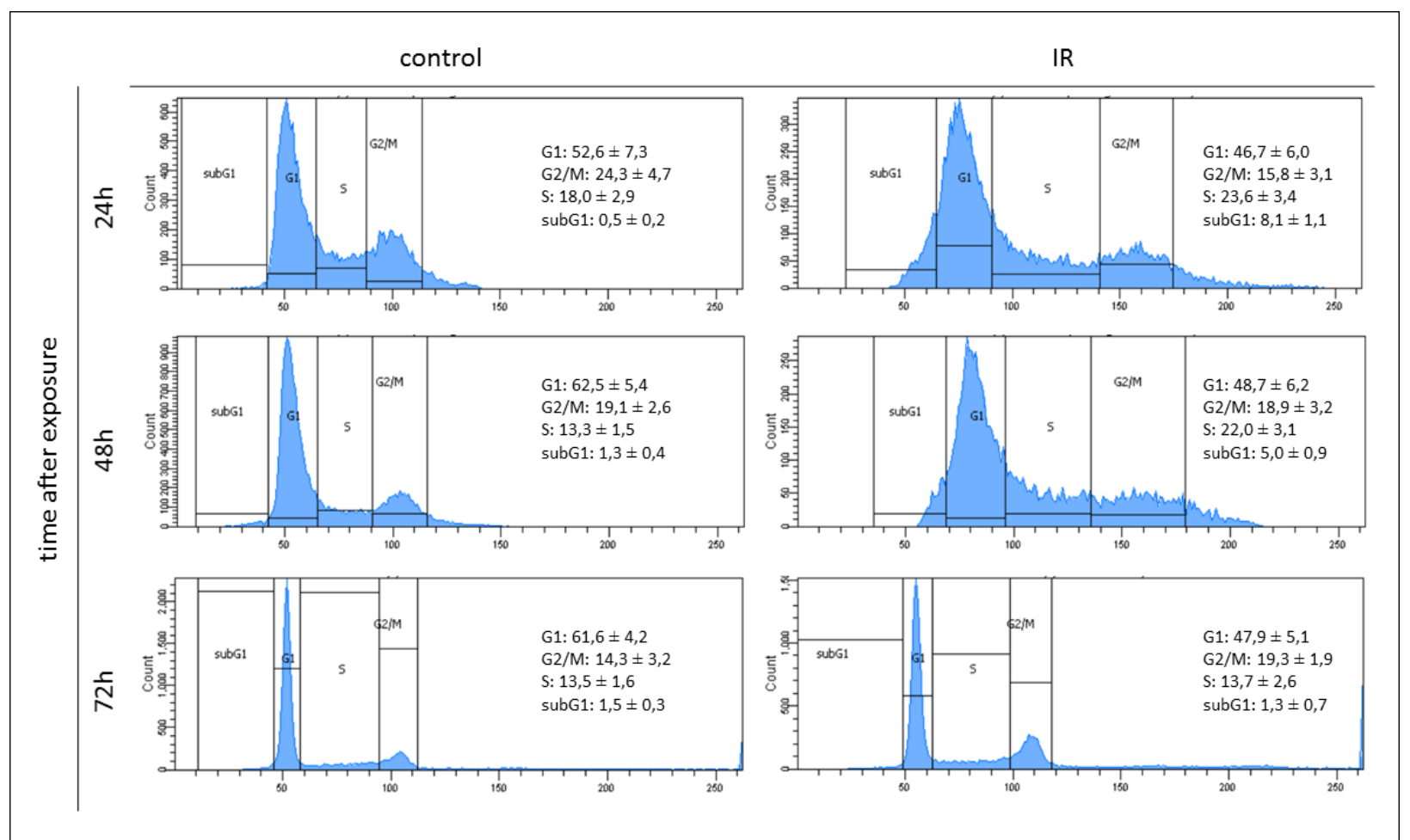

Figure 2. The influence of ionizing radiation on the distribution of the cell cycle phases.

The number of cells in different phases was counted by flow cytometry 1,2 and 3 days after the exposure to ionizing radiation and expressed as a percentage of all cells counted in a given sample. The average percentage of cells in subG1, G1, S and G2/M phases is shown in each diagram. 2 Gy radiation dose was used in each case. 


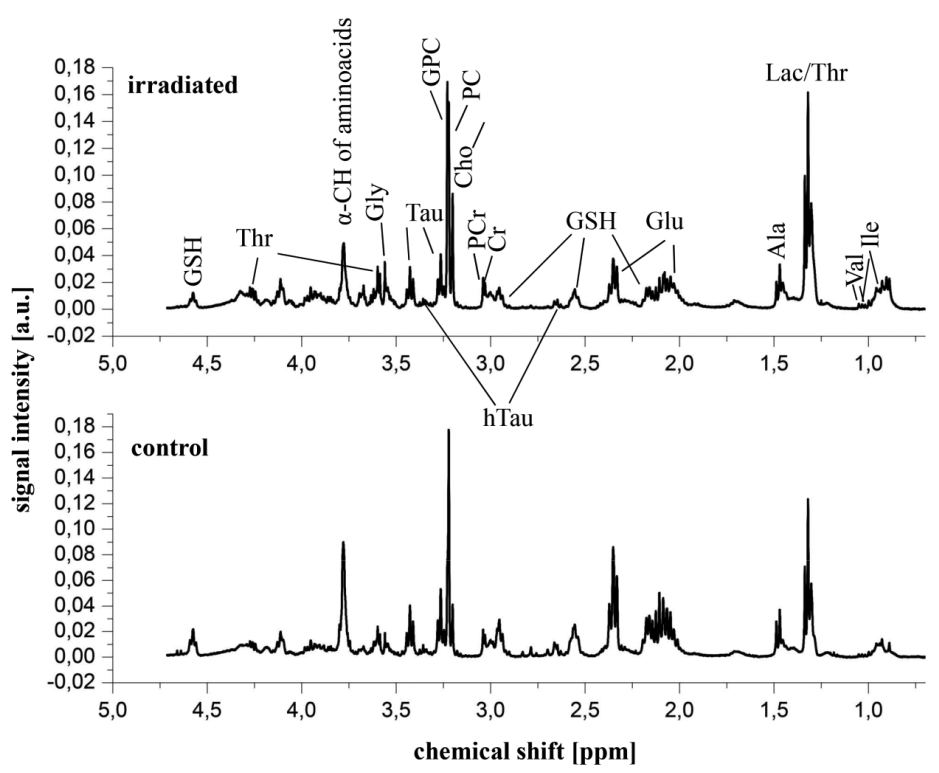

Figure 3. Representative ${ }^{1} \mathrm{H}$ HR-MAS NMR CPMG spectrum (after integral normalization) of control cells and cells $48 \mathrm{~h}$ after the exposure to ionizing radiation ( $2 \mathrm{~Gy}$ ).

GSH - glutathione; Thr - threonine; Gly - glycine; Tau - taurine; hTau - hypotaurine, GPC - glycerophosphocholine; PC - phosphocholine; Cho - choline; PCr - phosphocreatine; $\mathrm{Cr}$ - creatine; Glu - glutamate; Ala - alanine; Lac - lactate; Val - valine; lle - isoleucine.

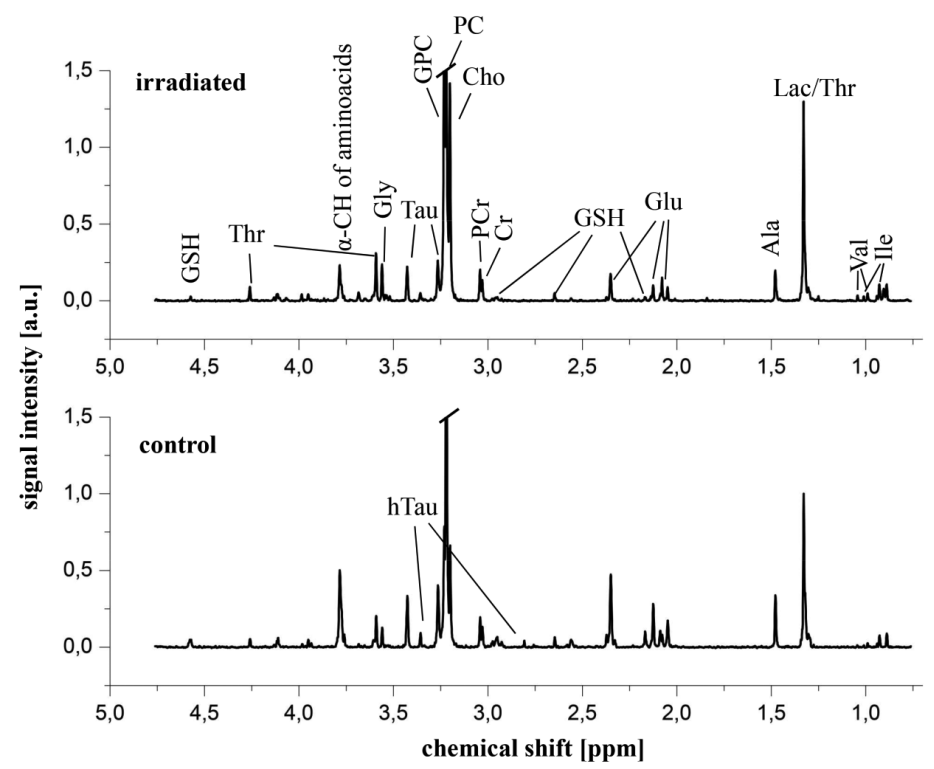

Figure 4. Representative ' $\mathrm{H}$ HR-MAS NMR p-Jres spectrum (after integral normalization) of the control cells and the cells $48 \mathrm{~h}$ after exposure to ionizing radiation ( $2 \mathrm{~Gy}$ ).

GSH - glutathione; Thr - threonine; Gly - glycine; Tau - taurine; hTau - hypotaurine; GPC - glycerophosphocholine; PC - phosphocholine; Cho - choline; PCr - phosphocreatine; $\mathrm{Cr}$ - creatine; Glu - glutamate; Ala - alanine; Lac - lactate; Val - valine; lle - isoleucine.

ate the metabolic changes the signals clearly representative of a single metabolite in the p-Jres or diffusion edited HR-MAS NMR spectra were analyzed.

Table 1 presents the metabolite levels (expressed either as peak areas for low molecular weight metabolites or as peak heights for lipids) in the irradiated and control cells as well as the change ratios calculated as the ratios of the signals' mean values for the irradiated cells and those for the control, non-irradiated ones (for both normalization methods, IN and PQN). As seen from these data, the main metabolic effect of cardiomyocytes' irradiation involved increase in threonine (Thr), glycine (Gly), free choline (Cho), glycerophosphocholine (GPC), valine ( Val), isoleucine (Ile) as well as lowering of the glutamate (Glu), reduced glutathione $(\mathrm{GSH})$, taurine (Tau) and hypotaurine (hTau) levels as compared to the appropriate values for the control samples. Both normalization methods reproduced the fold changes of these metabolites acceptably (especially regarding the direction of the changes). The main discrepancies concerned phosphocholine (PC), creatine (Cr) and phosphocreatine (PCr). For PC the integral normalization gave the fold change $<1$, whereas PQN yielded the value close to $1(1.09 \pm 0.10)$; the changes of $\mathrm{Cr}$ were visible only when PQN was applied (PQN showed the Cr level to increase following irradiation, whereas IN indicated no 
change in the Cr level), and, finally, though both normal- of radiation - considered often as small and harmless -

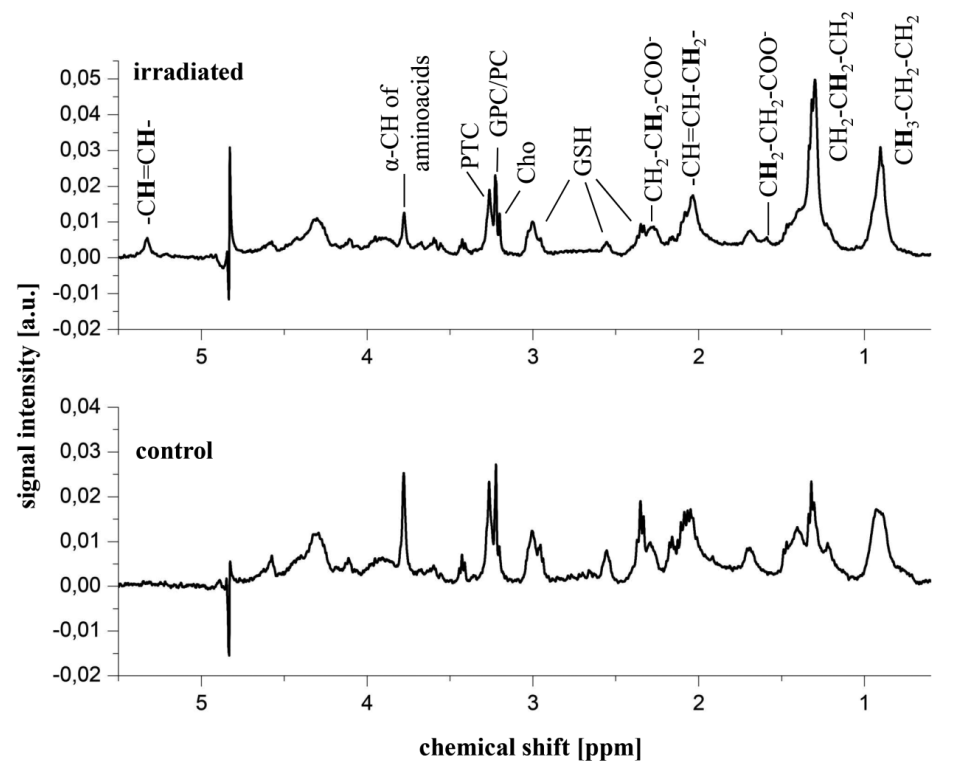

Figure 5. Representative ${ }^{1}$ H HR-MAS NMR diffusion edited spectrum (after integral normalization) of the control cells and the cells 48 $\mathrm{h}$ after exposure to ionizing radiation ( $2 \mathrm{~Gy})$.

PTC - phosphatidylcholine; GPC - glycerophosphocholine; PC - phosphocholine; Cho - choline; GSH - glutathione.

ization methods consistently showed an increase in $\mathrm{PCr}$ (Table 1), the uncertainties of the PCr levels were high. Thus, the changes in the normalized integrals of PC, $\mathrm{Cr}$ and PCr signals should be interpreted with caution. Nonetheless, the PCr/Cr, Cho/PC and GPC/PC ratios (Table 2) increased after the irradiation.

We also observed an increase in the lipid signals ( $\mathrm{Ta}$ ble 1), with the elevation of the $\mathrm{CH}_{2} / \mathrm{CH}_{3}$ ratio. Trypan blue staining showed that after NMR experiments the cells remained viable.

\section{DISCUSSION}

Metabolic effects of low-dose ionizing radiation on the heart cells have not been studied yet. However, many reports indicated that metabolic alterations occur in other human cells irradiated with low or high doses as well as under various pathological conditions (Kell et al., 2005; Barba \& Garcia-Dorado, 2012; Laiakis et al., 2012; Goudarzi et al., 2014; Kwon et al., 2014; Jang et al., 2016).

In this study, we showed that cardiomyocytes are relatively resistant to ionizing radiation, as the visible reduction of cell viability occurred only after doses higher than 4 Gy. Despite the small observed effect of relatively low doses ( $2 \mathrm{~Gy}$ ) of ionizing radiation on the cell viability, irradiation disturbed the distribution of cell cycle phases (block in the G2/M phase), induced apoptosis in cardiomyocytes. The intensity of apoptosis decreased in time and after an initial increase, the apoptotic subpopulation in the irradiated group was the same as in the control group. However, the G2/M cell cycle arrest in response to radiation could be a clear marker of the onset of cellular processes leading to DNA repair or apoptotic death (DiPaola, 2002).

The metabolic effects of radiation observed $48 \mathrm{~h}$ after the stimuli (the single dose of $2 \mathrm{~Gy}$ ) were detected using ${ }^{1} \mathrm{H}$ HR-MAS NMR and the results showed that the metabolic changes induced in cardiomyocytes by low doses are evident and may be related to the metabolites associated mainly with oxidative stress (glutamate, GSH, taurine), and with perturbations in multiple metabolic pathways, including energy metabolism (valine, isoleucine, GSH, glycine, threonine, taurine), membranes (phospholipids, cholines, glycine, taurine) and amino acids.

Oxidative stress is the main cause of ionizing radiation cardiotoxicity. Free radicals have the potential to damage all cell structures (proteins, lipids, nucleic acids), disrupt mitochondrial functioning and induce cardiac cell death and heart dysfunction (Khurana et al., 2013). Because of the destructive effect of free radicals, there are many pathways that allow cells to deal with oxidative stress and many metabolites are involved in these antioxidative reactions, including GSH, glutamate and taurine, whose concentrations changed after the radiation in our study.

Glutathione is one of the main antioxidants produced in cells in response to the oxidative stress (Shimzu et al., 1998; Grande et al., 2001; Khurana et al., 2013). However, it participates not only in antioxidant defense systems but also in many metabolic and detoxification processes (Lushchak, 2012). The latter function is also important because many toxic compounds are produced as side-products of the cellular metabolism - thus, the GSH depletion leaves the cells unprotected against the harmful compounds. Due to its cell roles, GSH has been accepted as an indicator of cell sensitivity to radiation (Grande et al., 2001; Santini et al., 2006a; Santini et al., 2006b; Palma et al., 2016). In our experiment, the GSH level in the irradiated cardiomyocytes was found to drop by 60 (PQN)-70\% (IN) as compared to the control cells (Table 1). Such loss of GSH may be due to its consumption during the oxidative stress induced by the irradiation, indicating a change in the antioxidant status of the cells, but at the same time, it may result from the inhibition of mitotic activity (Grande et al., 2001; Ladeirinha, 2011).

Taurine can be linked to glutathione by a common precursor - cysteine. Also, the final step of taurine biosynthesis typically requires the oxidation of hypotaurine 
Table 1. The list of the altered metabolite levels (expressed as peak heights for lipids and integrals for the remaining signals) obtained from the $p$-Jres and diffusion edited spectra.

\begin{tabular}{|c|c|c|c|c|c|c|}
\hline Metabolite & $\begin{array}{l}\text { Chemical } \\
\text { shift }\end{array}$ & $\begin{array}{l}\text { Normali } \\
\text { zation }\end{array}$ & $\begin{array}{l}\text { Irradiated } \\
\text { (mean } \pm S D)\end{array}$ & $\begin{array}{l}\text { Control } \\
(\text { mean } \pm S D)\end{array}$ & $\begin{array}{l}\text { Fold } \\
\text { change }\end{array}$ & $\begin{array}{l}\text { Spectrum } \\
\text { type }\end{array}$ \\
\hline \multirow{2}{*}{ Valine (Val) } & \multirow{2}{*}{1.04 ppm (d) } & IN & $0.19 \pm 0.06$ & $0.064 \pm 0.017$ & $2.94 \pm 1.75 \uparrow$ & \multirow{26}{*}{$\frac{\stackrel{u}{\alpha}}{\grave{\alpha}}$} \\
\hline & & PQN & $0.19 \pm 0.06$ & $0.046 \pm 0.010$ & $4.11 \pm 2.23 \uparrow$ & \\
\hline \multirow{2}{*}{ Isoleucine (Ile) } & \multirow{2}{*}{$1.01 \mathrm{ppm}(\mathrm{d})$} & IN & $0.164 \pm 0.004$ & $0.060 \pm 0.008$ & $2.74 \pm 0.41 \uparrow$ & \\
\hline & & PQN & $0.164 \pm 0.004$ & $0.043 \pm 0.003$ & $3.81 \pm 0.38 \uparrow$ & \\
\hline \multirow{2}{*}{ Glutamate (Glu) } & \multirow{2}{*}{$2.35 \mathrm{ppm}(\mathrm{m})$} & IN & $1.63 \pm 0.15$ & $5.31 \pm 0.15$ & $0.31 \pm 0.04 \downarrow$ & \\
\hline & & PQN & $1.63 \pm 0.15$ & $3.81 \pm 0.10$ & $0.43 \pm 0.05 \downarrow$ & \\
\hline \multirow{2}{*}{$\begin{array}{l}\text { Reduced } \\
\text { glutathione } \\
\text { (GSH) }\end{array}$} & \multirow{2}{*}{$2.17 \mathrm{ppm}(\mathrm{m})$} & IN & $0.30 \pm 0.12$ & $1.01 \pm 0.03$ & $0.30 \pm 0.13 \downarrow$ & \\
\hline & & PQN & $0.30 \pm 0.12$ & $0.73 \pm 0.06$ & $0.41 \pm 0.21 \downarrow$ & \\
\hline \multirow{2}{*}{$\begin{array}{l}\text { Phosphocholine } \\
\text { (PC) }\end{array}$} & \multirow{2}{*}{3.22 ppm (s) } & IN & $18.81 \pm 0.49$ & $23.81 \pm 0.40$ & $0.79 \pm 0.03 \downarrow$ & \\
\hline & & PQN & $18.81 \pm 0.49$ & $17.18 \pm 1.20$ & $1.09 \pm 0.10 \uparrow$ & \\
\hline \multirow{2}{*}{ Glycerophosphocholine (GPC) } & \multirow{2}{*}{3.23 ppm (s) } & IN & $17.70 \pm 2.38$ & $7.20 \pm 0.61$ & $2.45 \pm 0.54 \uparrow$ & \\
\hline & & PQN & $17.70 \pm 2.38$ & $5.21 \pm 0.71$ & $3.40 \pm 0.92 \uparrow$ & \\
\hline \multirow{2}{*}{ Choline (Cho) } & \multirow{2}{*}{3.20 ppm (s) } & IN & $13.61 \pm 0.83$ & $6.34 \pm 0.55$ & $2.14 \pm 0.32 \uparrow$ & \\
\hline & & PQN & $13.61 \pm 0.83$ & $4.58 \pm 0.64$ & $2.97 \pm 0.60 \uparrow$ & \\
\hline \multirow{2}{*}{ Threonine (Thr) } & \multirow{2}{*}{3.59 ppm (d) } & IN & $2.94 \pm 0.15$ & $1.87 \pm 0.24$ & $1.58 \pm 0.30 \uparrow$ & \\
\hline & & PQN & $2.94 \pm 0.15$ & $1.35 \pm 0.24$ & $2.17 \pm 0.51 \uparrow$ & \\
\hline \multirow{2}{*}{ Glicyne (Gly) } & \multirow{2}{*}{3.56 ppm (s) } & IN & $2.07 \pm 0.18$ & $0.89 \pm 0.12$ & $2.32 \pm 0.52 \uparrow$ & \\
\hline & & PQN & $2.07 \pm 0.18$ & $0.64 \pm 0.12$ & $3.21 \pm 0.89 \uparrow$ & \\
\hline \multirow{2}{*}{ Taurine (Tau) } & \multirow{2}{*}{3.43 ppm (t) } & IN & $2.37 \pm 0.26$ & $3.51 \pm 0.07$ & $0.68 \pm 0.09 \downarrow$ & \\
\hline & & PQN & $2.37 \pm 0.26$ & $2.53 \pm 0.09$ & $0.94 \pm 0.13 \downarrow$ & \\
\hline \multirow{2}{*}{ Hypotaurine (hTau) } & \multirow{2}{*}{2.65 ppm (t) } & IN & $0.32 \pm 0.07$ & $0.47 \pm 0.02$ & $0.68 \pm 0.19 \downarrow$ & \\
\hline & & PQN & $0.32 \pm 0.07$ & $0.340 \pm 0.003$ & $0.94 \pm 0.23 \leftrightarrow$ & \\
\hline \multirow{2}{*}{ Creatine $(\mathrm{Cr})$} & \multirow{2}{*}{3.02 ppm (s) } & IN & $1.13 \pm 0.05$ & $1.12 \pm 0.04$ & $1.00 \pm 0.08 \leftrightarrow$ & \\
\hline & & PQN & $1.13 \pm 0.05$ & $0.81 \pm 0.02$ & $1.39 \pm 0.09 \uparrow$ & \\
\hline \multirow{2}{*}{ Phosphocreatine (PCr) } & \multirow{2}{*}{3.03 ppm (s) } & IN & $1.68 \pm 0.05$ & $1.15 \pm 0.44$ & $1.46 \pm 0.60 \uparrow$ & \\
\hline & & PQN & $1.68 \pm 0.05$ & $0.84 \pm 0.36$ & $1.99 \pm 0.92 \uparrow$ & \\
\hline Phocnhatidvucholine (PTC) & $327 \mathrm{nnm}$ & IN & $2.17 \pm 0.10$ & $2.54 \pm 0.07$ & $0.85 \pm 0.06 \downarrow$ & \\
\hline 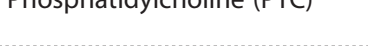 & 3.21 Ppm & PQN & $2.23 \pm 0.19$ & $2.48 \pm 0.13$ & $0.90 \pm 0.12 \downarrow$ & \\
\hline & $000 n m m$ & IN & $0.031 \pm 0.003$ & $0.016 \pm 0.001$ & $1.88 \pm 0.23 \uparrow$ & \\
\hline IIpla $\left(\mathrm{CH}_{3}-\mathrm{CH}_{2}-\mathrm{CH}_{2}\right)$ & $0.90 \mathrm{ppm}$ & PQN & $0.031 \pm 0.001$ & $0.016 \pm 0.001$ & $1.99 \pm 0.21 \uparrow$ & 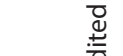 \\
\hline & $13 \mathrm{nnm}$ & IN & $0.050 \pm 0.005$ & $0.020 \pm 0.001$ & $2.49 \pm 0.39 \uparrow$ & $\bar{d}$ \\
\hline $\mathrm{H}_{2}^{-\mathrm{C}}$ & 1.3 ppm & PQN & $0.051 \pm 0.003$ & $0.020 \pm 0.002$ & $2.63 \pm 0.37 \uparrow$ & $\frac{\overline{0}}{\bar{s}}$ \\
\hline lini & $150 \mathrm{nnn}$ & IN & $0.0051 \pm 0.0004$ & $n=0$ & $n d$ & 蛋 \\
\hline 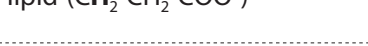 & 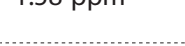 & PQN & $0.053 \pm 0.003$ & 11.u. & 11.u. & \\
\hline & $5.32 \mathrm{pnm}$ & IN & $0.0052 \pm 0.0005$ & & nd & \\
\hline$(-\mathrm{CH}=\mathrm{CH}-)$ & J.J2 Ppin & PQN & $0.0053 \pm 0.0004$ & & 11.u. & \\
\hline
\end{tabular}

The fold change was calculated as the ratio of the mean value (irradiated) to the mean value (control). n.d. - not detected in diffusion edited spectra, IN -integral normalization, PQN - probabilistic quotient normalization

to taurine. Both metabolites - hypotaurine and taurine - showed a similar decrease in this study (though, the value of the drop depended on the normalization method used, Table 1). Taurine is an amino acid accepted as an MR-detectable biomarker of apoptosis unrelated to necrosis (Rainaldi et al., 2008). In cardiac tissue, it promotes calcium transport, stabilizes cell membrane, regulates cellular osmolality, protects against oxidative stress and has its role in energy metabolism (Schaffer et al., 2010). The decrease in taurine in various types of irra- diated cells was described in the literature and associated with oxidative stress (Sokół et al., 2004; Santini et al., 2006b; Jang et al., 2016). There is a growing consensus that oxidative stress is linked to mitochondrial dysfunction (Ripps \& Shen, 2012). Thus, the total protective effect of taurine involves its antioxidant properties and improvement of mitochondrial function by stabilizing the electron transport chain and inhibiting the generation of reactive oxygen species. Due to the drop in taurine levels, the electron transport in respiratory chain is im- 
Table 2. Selected metabolite ratios determined from p-Jres spectra for irradiated and control samples.

\begin{tabular}{lcc}
\hline Metabolite ratio & Irradiated (mean \pm S.D.) & Control (mean \pm S.D.) \\
\hline Phosphocreatine / creatine (PCr/Cr) & $1.49 \pm 0.03$ & $1.03 \pm 0.43$ \\
\hline Choline / Phosphocholine (Cho/PC) & $0.72 \pm 0.03$ & $0.27 \pm 0.02$ \\
\hline Glycerophosphocholine / Choline (GPC/PC) & $0.94 \pm 0.15$ & $0.30 \pm 0.02$ \\
\hline
\end{tabular}

paired leading to an increase of oxidative stress and the decrease of ATP synthesis (Jong et al., 2012). Reduced ATP levels in hypoxic cells are also claimed to contribute to the decrease in choline phosphorylation (Bansal et al., 2012). In our experiment, however, the variations of phosphocholine observed after irradiation (Table 1) are too ambiguous to draw conclusions from them (with PQN there is no change in the integral intensity of this signal). Also, the analysis of the $\mathrm{PCr}-\mathrm{Cr}$ relationships - important, because PCr (and $\mathrm{Cr}$ ) is involved in oxidative stress protection - did not provide a direct clue concerning the energetic processes (due to the high uncertainties of the PCr fold changes and the ambiguous $\mathrm{Cr}$ fold changes). It can be only speculated that because the $\mathrm{PCr} / \mathrm{Cr}$ ratio bears a predictable relationship to the $\mathrm{ATP} / \mathrm{ADP}$ ratio in the cell, the increase in $\mathrm{PCr} / \mathrm{Cr}$ measured 48 hours post irradiation ( $\mathrm{PCr} / \mathrm{Cr}$ is $\sim 1.5$ for the irradiated cells, whereas for the control samples it is ca. 1 , regardless of the normalization method applied) points rather towards a regeneration of the cellular ATP level (Saks et al., 2006) with a supranormal restoration of PCr. Such "PCr overshoot phenomenon" associated with a slower and often incomplete ATP recovery after a period of ischemia (or anoxia) was reported to occur in the reperfused myocardium (Kida et al., 1993). However, the $\mathrm{Cr}-\mathrm{PCr}$ energetic mechanism is so complex and depends on so many factors, that a separate study is required to investigate the effects of hypoxia and stress onto these dependencies. It is reflected, for instance, by the dynamic changes in the integral intensities of the creatine and phosphocreatine signals - as measured in vivo - during the irradiation cycle and long after it; these changes are accompanied by the variations in the T1 relaxation times (Sokół et al., 2004).

Taurine content can be dramatically altered by osmotic stress: cardiomyocytes exposed to hypoosmotic conditions undergo cell swelling, followed by extrusion of osmolytes, such as taurine, from the cell, whereas the regulatory response to hyperosmotic conditions leads to taurine influx into the cell (Thurston et al, 1981); this mechanism may lead to cell deformation and shrinkage, which was reported for several cell types as characteristic for apoptosis (Lang et al., 2000; Morán et al., 2000; Friis et al., 2005). Taurine deficiency itself may trigger apoptosis through an initiation of the mitochondrial permeability transition, which is dependent on both calcium overload and oxidative stress (Schaffer et al., 2010).

The NMR studies on metabolic alterations in apoptotic cells - HL60 promyelocytic leukemia cells (Milkevitch et al., 2005) and DU145 human prostatic carcinoma cells (Rainaldi et al., 2008) - reported decreased levels of choline, glutamate, GSH and taurine, and increased levels of mobile lipids. The metabolic pattern obtained in this study provided similar results for irradiated cardiomyocytes, however, different for total choline. In the irradiated cells, we observed free choline and glycerophosphocholine (GPC) to be markedly increased (two or three times), and phosphatidylcholine to be decreased by 10 15\% (Table 1). Choline, the major membrane constituent, and PC are precursors of phosphatidylcholine, while glycerophosphocholine is a choline derivative and one of the two major forms of choline storage (along with PC) formed from phosphatidylcholine. Unlike GPC, phosphocholine is both catabolic and anabolic metabolite: its increase may indicate its de novo biosynthesis from free choline but also membrane disruption, as it is phosphatidylcholine breakdown product. The decrease in the signal intensity of phosphocholine may indicate induction of growth arrest that takes place during the onset of apoptosis (Bezabeh et al., 2001). However, in case of any perturbation in cell membrane metabolism GPC and PC may change and their mutual changes are of importance. The disturbed choline metabolism and proportions of GPC/PC and Cho/PC observed here in irradiated cardiomyocytes were described in literature as common effect of ionizing radiation (Grande et al., 2001; Sokół et al., 2004; Santini et al., 2006a; Santini et al., 2006b; Jang et al., 2016). High levels of choline can cause an increase in glycine level. In fact, we observed 2-3 fold Gly increase in the irradiated cardiomyocytes (Table 1). We also performed some pilot experiments using GC-MS to analyze metabolites extracted from irradiated cardiomyocytes and found an about 2.5-fold increase in abundance of glycine, which confirmed the results received with NMR. Glycine is a catabolic product of choline (Locasale, 2013) and acts as a precursor for several key metabolites of low molecular weight, e.g. glutathione.

The results of this work indicate that phospholipid metabolism is influenced by the ionizing radiation and the effects observed by ${ }^{1} \mathrm{H}$ MAS NMR are due to a damage of membrane structures - among others mitochondria - in the cells. Such supposition is supported by the available literature (Santini et al., 2006a; Barba et al., 2007; Kwon et al., 2014, Palma et al., 2016). We observed an increase in the signals arising from methylene (-CH2- $\left.\mathrm{CH}_{2}-\mathrm{CH} 2-\right)$ and methyl $\left(\mathrm{CH}_{3}-\mathrm{CH} 2-\right)$ groups at 1.3 and $0.9 \mathrm{ppm}$, respectively. The $\mathrm{CH}_{2} / \mathrm{CH}_{3}$ ratio increased from 1.25 (non-irradiated cells) to 1.61 (irradiated cells). Increase in the lipid signals, especially increase in $\mathrm{CH}_{2}$ / $\mathrm{CH}_{3}$ ratio, is frequently claimed to be associated with progression of apoptotic death (Santini et al., 2006a; Santini et al., 2006b; Rainaldi et al., 2008; Matulewicz et al., 2013) and with disruption of fatty acid metabolism (Goudarzi et al., 2014). Blankenberg et al. (Blankenberg et al., 1997) observed that in lymphoblasts treated with doxorubicin the onset of apoptosis was accompanied by over twofold increase in the $\mathrm{CH}_{2}$ resonance. They suggested that increased mobility of the plasma membrane lipids may be due to a loss of cytoskeletal architecture and the $\mathrm{CH}_{2} / \mathrm{CH}_{3}$ ratio could be proportional to the percentage of apoptotic cells in vitro.

Along with methylene and methyl groups signals increase the lipid signal at $5.32 \mathrm{ppm}$ increased markedly after irradiation (Table 1). It corresponded to ceramide, a sphingolipid that is a major membrane component. Perry and coworkers (Perry et al., 2000) showed its increase in etoposide-induced apoptosis of Molt-4 human leukemia cells and suggested that ceramide functions as a regulatory molecule in mediating membrane related apoptotic events. The increase in ceramide, with its long 
$-\mathrm{CH}_{2}$-chain, could also explain the increase in the 1.3 $\mathrm{ppm}$ signal intensity in our NMR spectra. Increasing levels of phospholipids and choline compounds after the exposure to radiation indicate that the cell membrane was damaged, presumably by free radical ions induced by radiation (Kwon et al., 2014). It is also possible that the changes in phospholipid profiles are correlated with hypoxic conditions (Lin et al., 2009; Palma et al., 2016) and many studies reported the initiation of lipid peroxidation following the radiation exposure (Pathak et al., 2007; Jang et al., 2016).

According to existing literature heart muscle cells (cardiomyocytes) have very high energy demands. Consequently, proper heart functioning depends on the presence of adequate amounts of oxygen and oxidizable substrates for energy production in order to meet the demands of working heart (Carvajal et al., 2003; Zervou et al., 2016). Mitochondria, which occupy $30 \%$ of the cardiac cell volume, produce $90 \%$ of ATP - a source of chemical energy in the cell - and ATP is generated almost exclusively from the aerobic metabolism of glucose, lactate, and fatty acids coupled to mitochondrial oxidative phosphorylation (Carvajal \& Moreno-Sánchez, 2003). Any disruption of metabolic pathways that reduces the energy production can result in dysfunction of cardiomyocytes and the whole heart. Among the changes observed by us in metabolic profiles of the irradiated cells there were also those involved in energetic pathways: alteration of threonine and glycine level can be associated with disruption of the citric acid cycle (Andreadou et al., 2009; Jang et al., 2016), while increase in lipids indicates impaired beta-oxidation and is described in the literature as a radiation effect (Goudarzi et al., 2014; Palma et al., 2016). Disruption of these two energetic pathways: beta-oxidation, which produces acetyl-CoA, and citric acid cycle, converting acetyl-CoA to energy, can result in insufficient energy production and lead to cardiomyocytes' death.

Leucine, isoleucine and valine, the three branchedchain amino acids (BCAAs), play an important role in metabolic and physiological processes (Harris et al., 2004; Tom \& Nair, 2006). Two of them: isoleucine and valine increased distinctly (3-4 folds). The radiation-induced increase of these amino acids can result from protein breakdown or DNA damage (Schwenen et al., 1989). An increase of isoleucine and valine was observed in the plasma after doxorubicin treatment and in the spleen after ionizing radiation and is probably connected with oxidative stress (Schnackenberg et al., 2016; Xiao et al., 2016). Radiation-induced disruption of these amino acids metabolism may be associated with radiation ability to affect energy and fatty acid metabolism (Goudarzi et al., 2014).

Many authors claim that metabolomics is very sensitive to environmental or genetic interventions, and provides one of the most effective methods of early detection of metabolic changes in cells, tissues or body fluids. Our results seem to be consistent with such statement - the NMR analysis enabled us to detect the metabolic changes in irradiated cells, while standard biological tests revealed to be less sensitive. Thus, NMR opens new possibilities for monitoring changes in metabolites associated with heart damage, and what is more important, for identifying the metabolites that change depending on the toxic agent applied (Barba \& Garcia-Dorado, 2012). Several studies showed that various cardiac disorders are represented by different metabolic biomarkers. For example, in case of heart ischemia lactate metabolism is disturbed, in case of cardiomyopathy - creatine is the main biomarker, and in infarcted areas of the heart - lipid compounds are of interest (Nakae et al., 2003; Faller et al., 2013).

The effect of low-dose radiation on the cardiac metabolic profile should be further analyzed using animal models. The results obtained in this research, using cultured cardiomyocytes, may differ from those from animal models, primarily due to the complexity of the interactions in the living organism. Nevertheless, our results strongly indicate that ionizing radiation affects cardiac cells metabolome and HR-MAS NMR technique is suitable and sufficiently sensitive for their detection.

In conclusion, the problem of cardiotoxicity of lowdose radiation has become more serious because of a high risk of exposure and because of increasing life expectancy, which allows for revealing of long-term effects of radiation. Detailed analysis of the metabolic changes observed in this study suggests that low-dose radiation initiates cell death program in cardiomyocytes and processes invoked by radiation may lead to cardiotoxicity. Our results showed that ionizing radiation induces oxidative stress, affects energetic pathways and damages membrane structures of cardiomyocytes, and can eventually lead to cell death even at low doses, which potentially have no effect on cell viability. Identification of metabolites disturbed by low-dose radiation in the heart cells may allow early detection of changes leading to the development of cardiovascular diseases and taking steps to prevent them.

\section{Conflict of interest}

The authors declare that they have no conflict of interest.

\section{Acknowledgements of Financial Support}

This work was supported by the National Science Centre, Grant 2015/17/N/NZ7/04101.

\section{REFERENCES}

Andreadou I, Papaefthimiou M, Zira A, Constantinou M, Sigala F, Skaltsounis AL, Tsantili-Kakoulidou A, Iliodromitis EK, Kremastinos DT, Mikros E (2009) Metabonomic identification of novel biomarkers in doxorubicin cardiotoxicity and protective effect of the natural antioxidant oleuropein. NMR Biomed 22: 585-592. doi: $10.1002 / \mathrm{nbm} .1370$

Bansal A, Harris RA, DeGrado TR (2012) Choline phosphorylation and regulation of transcription of choline kinase $\alpha$ in hypoxia. $J \mathrm{~L} i$ pid Res 53: 149-157. doi: 10.1194/jlr.M021030

Barba I, Garcia-Dorado D (2012) Metabolomics in cardiovascular disease: towards clinical application. In Coronary Artery Disease - New Insights and Novel Approaches. pp 207-224, InTech

Barba I, Jaimez-Auguets E, Rodriguez-Sinovas A, Garcia-Dorado D (2007) 1H NMR-based metabolomic identification of at-risk areas after myocardial infarction in swine. MAGMA 20: 265-271

Bezabeh T, Mowat MR, Jarolim L, Greenberg AH, Smith IC (2001) Detection of drug-induced apoptosis and necrosis in human cervical carcinoma cells using $1 \mathrm{H}$ NMR spectroscopy. Cell Death Differ 8: 219-224. doi: 10.1038/sj.cdd.4400802

Blankenberg FG, Katsikis PD, Storrs RW, Beaulieu C, Spielman D, Chen JY, Naumovski L, Tait JF (1997) Quantitative analysis of apoptotic cell death using proton nuclear magnetic resonance spectroscopy. Blood 89: 3778-3786

Boerma M, Sridharan V, Mao XW, Nelson GA, Cheema AK, Koturbash I, Singh SP, Tackett AJ, Hauer-Jensen M (2016) Effects of ionizing radiation on the heart. Mutat Res 770: 319-327. doi: 10.1016/j.mrrev.2016.07.003

Bouillon K, Haddy N, Delaloge S, Garbay JR, Garsi JP, Brindel P, MousannifA, Lê MG, Labbe M, Arriagada R, Jougla E, Chavaudra J, Diallo I, Rubino C, de Vathaire F (2011) Long-term cardiovascular mortality after radiotherapy for breast cancer. $J$ Am Coll Cardiol 57: 445-452. doi: 10.1016/j.jacc.2010.08.638 
Carvajal K, Moreno-Sánchez R (2003) Heart metabolic disturbances in cardiovascular diseases. Arch Med Res 34: 89-99. doi: 10.1016/ S0188-4409(03)00004-3

Craig A, Cloarec O, Holmes E, Nicholson JK, Lindon JC (2006) Scaling and normalization effects in NMR spectroscopic metabonomic data sets. Anal Chem 78: 2262-2267

Darby SC, Ewertz M, McGale P, Bennet AM, Blom-Goldman U, Brønnum D, Correa C, Cutter D, Gagliardi G, Gigante B, Jensen MB, Nisbet A, Peto R, Rahimi K, Taylor C, Hall P (2013) Risk of ischemic heart disease in women after radiotherapy for breast cancer. N Engl J Med 368: 987-998. doi: 10.1056/NEJMoa1209825

Delp MD, Charvat JM, Limoli CL, Globus RK, Ghosh P (2016) Apollo lunar astronauts show higher cardiovascular disease mortality: possible deep space radiation effects on the vascular endothelium. Sci Rep 6: 29901. doi: 10.1038/srep29901

Dieterle F, Ross A, Schlotterbeck G, Senn H (2006) Probabilistic quotient normalization as robust method to account for dilution of complex biological mixtures. application in $1 \mathrm{H}$ NMR metabonomics. Anal Chem 78: 4281-4290

DiPaola RS (2002) To arrest or not to G(2)-M Cell-cycle arrest: commentary re: A. K. Tyagi et al., Silibinin strongly synergizes human prostate carcinoma DU145 cells to doxorubicin-induced growth inhibition, G(2)-M arrest, and apoptosis (2002) Clin Cancer Res 8: 3512-3519, Clin Cancer Res 8: 3311-3314

Eckhart AD, Beebe K, Milburn M (2012) Metabolomics as a key integrator for "omic" advancement of personalized medicine and future therapies. Clin Transl Sci 5: 285-288. doi: 10.1111/j.17528062.2011.00388.x

Evans KM, Bodmer J, Edwards B, Levins J, O'Meara A, Ruhotina M, Smith R, Delaney T, Hoffman-Contois R, Boccuzzo L, Hales H, Carney JK (2015) An Exploratory analysis of public awareness and perception of ionizing radiation and guide to public health practice in Vermont. I Environ Public Health 2015: 476495. doi: $10.1155 / 2015 / 476495$

Faller KM, Lygate CA, Neubauer S, Schneider JE (2013) (1)H-MR spectroscopy for analysis of cardiac lipid and creatine metabolism. Heart Fail Rev 18: 657-668. doi: 10.1007/s10741-012-9341-z

Friis MB, Friborg CR, Schneider L, Nielsen MB, Lambert IH, Christensen ST, Hoffmann EK (2005) Cell shrinkage as a signal to apoptosis in NIH 3T3 fibroblasts. J Physiol 567 (Pt 2): 427-443. doi: 10.1113/jphysiol.2005.087130

Goudarzi M, Mak TD, Chen C, Smilenov LB, Brenner DJ, Fornace AJ (2014) The effect of low dose rate on metabolomic response to radiation in mice. Radiat Environ Biophys 53: 645-657. doi: 10.1007/ s00411-014-0558-1

Grande S, Luciani AM, Rosi A, Cherubini R, Conzato M, Guidoni L, Viti V (2001) Radiation effects on soluble metabolites in cultured HeLa cells examined by $1 \mathrm{H}$ MRS: changes in concentration of glutathione and of lipid catabolites induced by gamma rays and proton beams. Int J Cancer 96 (Suppl): 27-42. doi: 10.1002/ijc.10345

Gu Y, Mi W, Ge Y, Liu H, Fan Q, Han C, Yang J, Han F, Lu X, $\mathrm{Yu}$ W (2010) GlcNAcylation plays an essential role in breast cancer metastasis. Cancer Res 70: 6344-6351. doi: 10.1158/0008-5472. CAN-09-1887

Harris RA, Joshi M, Jeoung NH (2004) Mechanisms responsible for regulation of branched-chain amino acid catabolism. Biochem Biophys Res Commun 313: 391-396

Jang WG, Park JY, Lee J, Bang E, Kim SR, Lee EK, Yun HJ, Kang CM, Hwang GS (2016) Investigation of relative metabolic changes in the organs and plasma of rats exposed to X-ray radiation using HR-MAS (1)H NMR and solution (1)H NMR. NMR Biomed 29: 507-518. doi: $10.1002 / \mathrm{nbm} .3485$

Jong CJ, Azuma J, Schaffer S (2012) Mechanism underlying the antioxidant activity of taurine: prevention of mitochondrial oxidant production. Amino Acids 42: 2223-2232. doi: 10.1007/s00726-0110962-7

Kell DB, Brown M, Davey HM, Dunn WB, Spasic I, Oliver SG (2005) Metabolic footprinting and systems biology: the medium is the message. Nat Rev Microbiol 3: 557-565

Khurana S, Hollingsworth A, Piche M, Venkataraman K, Kumar A, Ross GM, Tai TC (2014) Antiapoptotic actions of methyl gallate on neonatal rat cardiac myocytes exposed to H2O2. Oxid Med Cell Longev 2014: 657512 doi: 10.1155/2014/657512

Kida M, Fujiwara H, Uegaito T, Miyamae M, Ohura M, Miura I, Yabuuchi Y (1993) Dobutamine prevents both myocardial stunning and phosphocreatine overshoot without affecting ATP level. I Mol Cell Cardiol 25: 875-885

Kwon YK, Ha IJ, Bae HW, Jang WG, Yun HJ, Kim SR, Lee EK, Kang CM, Hwang GS (2014) Dose-dependent metabolic alterations in human cells exposed to gamma irradiation. PLoS One 9: e113573. doi: 10.1371 /journal.pone. 0113573

Ladeirinha AFF (2011) Establishment of primary cell cultures from lung tissue biopsies and study of the cellular metabolic responses to cisplatin and radiation exposure. Disseration, Universidade de Coimbra
Laiakis EC, Hyduke DR, Fornace AJ (2012) Comparison of mouse urinary metabolic profiles after exposure to the inflammatory stressors $\gamma$ radiation and lipopolysaccharide. Radiat Res 177: 187-199

Lang F, Ritter M, Gamper N, Huber S, Fillon S, Tanneur V, LeppleWienhues A, Szabo I, Gulbins E (2000) Cell volume in the regulation of cell proliferation and apoptotic cell death. Cell Physiol Biochem 10: $417-428$

Lin H, Zhang J, Gao P (2009) Silent myocardial ischemia is associated with altered plasma phospholipids. J Clin Lab Anal 23: 45-50. doi: $10.1002 /$ jcla. 20288

Little MP, Azizova TV, Bazyka D, Bouffler SD, Cardis E, Chekin S, Chumak VV, Cucinotta FA, de Vathaire F, Hall P, Harrison JD, Hildebrandt G, Ivanov V, Kashcheev VV, Klymenko SV, Kreuzer M, Laurent O, Ozasa K, Schneider T, Tapio S, Taylor AM, Tzoulaki I, Vandoolaeghe WL, Wakeford R, Zablotska LB, Zhang W, Lipshultz SE (2012) Systematic review and meta-analysis of circulatory disease from exposure to low-level ionizing radiation and estimates of potential population mortality risks. Environ Health Perspect 120: 1503-1511. doi: 10.1289/ehp.1204982

Locasale JW (2013) Serine, glycine and one-carbon units: cancer metabolism in full circle. Nat Rev Cancer 13: 572-583. doi: 10.1038/ nrc3557

Ludwig C, Viant MR (2010) Two-dimensional J-resolved NMR spectroscopy: review of a key methodology in the metabolomics toolbox. Phytochem Anal 21: 22-32. doi: 10.1002/pca.1186

Lushchak VI (2012) Glutathione homeostasis and functions: potential targets for medical interventions. J Amino Acids 2012: 736837. doi: 10.1155/2012/736837

Matulewicz Ł, Cichoń A, Sokół M, Przybyszewski W, GłowalaKosińska M, Gibas M (2013) High resolution proton nuclear magnetic resonance (1H NMR) spectroscopy of surviving C6 glioma cells after X-ray irradiation. Folia Neuropathol 51: 33-43

Milkevitch M, Shim H, Pilatus U, Pickup S, Wehrle JP, Samid D, Poptani H, Glickson JD, Delikatny EJ (2005) Increases in NMR-visible lipid and glycerophosphocholine during phenylbutyrate-induced apoptosis in human prostate cancer cells. Biochim Biophys Acta 1734: 1-12. doi: 10.1016/j.bbalip.2005.01.008

Monceau V, Meziani L, Strup-Perrot C, Morel E, Schmidt M, Haagen J, Escoubet B, Dörr W, Vozenin MC (2013) Enhanced sensitivity to low dose irradiation of ApoE-/- mice mediated by early proinflammatory profile and delayed activation of the TGF 11 cascade involved in fibrogenesis. PLoS One 8: e57052. doi: 10.1371/journal. pone.0057052

Morán J, Hernández-Pech X, Merchant-Larios H, Pasantes-Morales H (2000) Release of taurine in apoptotic cerebellar granule neurons in culture. Pflugers Arch 439: 271-277

Nakae I, Mitsunami K, Omura T, Yabe T, Tsutamoto T, Matsuo S, Takahashi M, Morikawa S, Inubushi T, Nakamura Y et al (2003) Proton magnetic resonance spectroscopy can detect creatine depletion associated with the progression of heart failure in cardiomyopathy. J Am Coll Cardiol 42: 1587-1593

Palma A, Grande S, Luciani AM, Mlynárik V, Guidoni L, Viti V, Rosi A (2016) Metabolic study of breast MCF-7 tumor spheroids after gamma irradiation by (1)H NMR spectroscopy and microimaging. Front Oncol 6: 105. doi: 10.3389/fonc.2016.00105

Pan X, Wilson M, Mirbahai L, McConville C, Arvanitis TN, Griffin JL, Kauppinen RA, Peet AC (2011) In vitro metabonomic study detects increases in UDP-GlcNAc and UDP-GalNAc, as early phase markers of cisplatin treatment response in brain tumor cells. I Proteome Res 10: 3493-3500. doi: 10.1021/pr200114v

Pathak CM, Avti PK, Kumar S, Khanduja KL, Sharma SC (2007) Whole body exposure to low-dose gamma radiation promotes kidney antioxidant status in Balb/c mice. I Radiat Res 48: 113-120

Perry DK, Carton J, Shah AK, Meredith F, Uhlinger DJ, Hannun YA (2000) Serine palmitoyltransferase regulates de novo ceramide generation during etoposide-induced apoptosis. J Biol Chem 275: $9078-$ 9084

Prosnitz RG, Hubbs JL, Evans ES, Zhou SM, Yu X, Blazing MA, Hollis DR, Tisch A, Wong TZ, Borges-Neto S, Hardenbergh PH, Marks LB (2007) Prospective assessment of radiotherapy associated cardiac toxicity in breast cancer patients: analysis of data 3 to 6 years after treatment. Cancer 110: 1840-1850

Rainaldi G, Romano R, Indovina P, Ferrante A, Motta A, Indovina PL, Santinia MT (2008) Metabolomics using 1H-NMR of apoptosis and Necrosis in HL60 leukemia cells: differences between the two types of cell death and independence from the stimulus of apoptosis used. Radiat Res 169: 170-180. doi: 10.1667/RR0958.1

Rhee EP, Gerszten RE (2012) Metabolomics and cardiovascular biomarker discovery. Clin Chem 58: 139-147. doi: 10.1373/ clinchem.2011.169573

Ripps H, Shen W (2012) Review: taurine: a "very essential" amino acid. Mol Vis 18: 2673-2686

Saks V, Dzeja P, Schlattner U, Vendelin M, Terzic A, Wallimann T (2006) Cardiac system bioenergetics: metabolic basis of the FrankStarling law. J Physiol 571 (Pt 2): 253-273. doi:10.1113/jphysiol.2005.101444.Santini MT, Romano R, Rainaldi G, Ferrante A, In- 
dovina P, Motta A, Indovina PL (2006a) 1H-NMR evidence for a different response to the same dose (2 Gy) of ionizing radiation of MG-63 human osteosarcoma cells and three-dimensional spheroids. Anticancer Res 26: 267-281

Santini MT, Romano R, Rainaldi G, Indovina P, Ferrante A, Motta A, Indovina PL (2006b) Temporal dynamics of 1H-NMR-visible metabolites during radiation-induced apoptosis in MG-63 human osteosarcoma spheroids. Radiat Res 166: 734-745. doi: 10.1667/ RR0635.1

Schaffer SW, Jong CJ, Ramila KC, Azuma J (2010) Physiological roles of taurine in heart and muscle. J Biomed Sci 17 (Suppl 1): S2. doi: 10.1186/1423-0127-17-S1-S2

Schnackenberg LK, Pence L, Vijay V, Moland CL, George N, Cao Z, Yu LR, Fuscoe JC, Beger RD, Desai VG (2016) Early metabolomics changes in heart and plasma during chronic doxorubicin treatment in B6C3F1 mice. J Appl Toxicol 36: 1486-1495. doi: $10.1002 /$ jat.3307

Schwenen M, Altman KI, Schröder W (1989) Radiation-induced increase in the release of amino acids by isolated, perfused skeletal muscle. Int J Radiat Biol 55: 257-269

Shimzu T, Iwanaga M, Yasunaga A, Urata Y, Goto S, Shibata S, Kondo T (1998) Protective role of glutathione synthesis on radiation-induced DNA damage in rabbit brain. Cell Mol Neurobiol 18: 299-310

Shimizu Y, Kodama K, Nishi N, Kasagi F, Suyama A, Soda M, Grant EJ, Sugiyama H, Sakata R, Moriwaki H, Hayashi M, Konda M,
Shore RE (2010) Radiation exposure and circulatory disease risk: Hiroshima and Nagasaki atomic bomb survivor data, 1950-2003. BMJ 340: b5349. doi: 10.1136/bmj.b5349

Sokół M, Przybyszewski WM, Matlas B (2004) Investigation of metabolic changes in irradiated rat brain tissue by means of $1 \mathrm{H}$ NMR in vitro relaxation study. Solid State Nucl Magn Reson 25: 53-60. doi: 10.1016/i.ssnmr.2003.03.013

Thurston JH, Hauhart RE, Naccarato EF (1981) Taurine: possible role in osmotic regulation of mammalian heart. Science 214: 1373-1374

Tom A, Nair KS (2006) Assessment of branched-chain amino Acid status and potential for biomarkers. J Nutr 136 (1 Suppl): 324S$330 \mathrm{~S}$

Vasconcelos-Dos-Santos A, Oliveira IA, Lucena MC, Mantuano NR, Whelan SA, Dias WB, Todeschini AR (2015) Biosynthetic machinery involved in aberrant glycosylation: promising targets for developing of drugs against cancer. Front Oncol 5: 138. doi: 10.3389/ fonc.2015.00138

Wells L, Vosseller K, Hart GW (2003) A role for N-acetylglucosamine as a nutrient sensor and mediator of insulin resistance. Cell Mol Life Sci 60: 222-228

Xiao X, Hu M, Liu M, Hu JZ (2016) 1H NMR Metabolomics study of spleen from C57BL/6 mice exposed to gamma radiation. Metabolomics (Los Angel) 6: 1-11. doi: 10.4172/2153-0769.1000165

Zervou S, Whittington HJ, Russell AJ, Lygate CA (2016) Augmentation of creatine in the heart. Mini Rev Med Chem 16: 19-28 